\title{
Development of high concentrated aqueous silver nanofluid and inkjet printing on ceramic substrates
}

\author{
A. Kosmala, Q. Zhang*, R. Wright and P. Kirby \\ Department of Materials, Cranfield University, Cranfield, Bedfordshire, MK43 0AL, UK \\ *Author for correspondence q.zhang@ cranfield.ac.uk
}

\begin{abstract}
In this paper, the formulation of a high Ag loading (45 wt \%) aqueous ink and its subsequent printing on three different ceramic substrates were reported. Monodispersed Ag nanoparticles with a size down to $10 \mathrm{~nm}$ were successfully synthesized in aqueous medium. These nanoparticles were then successfully dispersed up to $45 \mathrm{wt} \%$ in aqueous medium with the aid of a co-polymer, Pluronic F127. The printed tracks show the electrical conductivity of $3 \mu \Omega \mathrm{cm}$ close to the value of silver bulk $(1.6 \mu \Omega \mathrm{cm})$. The use of high solid loading inks reduces the number of printed layers required for thick, dense and conductive film thus leading to the reduction of the costs, and high efficiency of the printing process. High solid loading also results in the finer printed features. The effect of substrates, printing temperature and dot spacing on the size and morphology of printed silver features was investigated. Increasing the dot spacing together with the substrates temperature resulted in the limited ink spreading, hence narrow printed line and improved geometry of printed patterns.
\end{abstract}

\section{Introduction}

Direct printing of circuits onto substrates is a key technology for driving down the cost of micro-manufacturing. In the continuing demand for finer feature sizes the inkjet printing has emerged as an attractive option for bridging the gap between high resolution $(1 \mu \mathrm{m}$ and below) which is likely to remain the province of conventional lithography, and the $\sim 100 \mu \mathrm{m}$ resolution achievable with for example screen printing. The printing of metal inks, especially those containing silver nanoparticles (Ag NPs), has been found to be a very powerful tool for 
direct patterning of electrically conductive structures and low-resistance interconnects in electronic devices [1-3].

The ceramics play an important role in electronics miniaturization and have potential to become a mass production technology for non-silicon microsystems. Using ceramic tapes it is possible to integrate small passive components on ceramic substrates in 3-D that give considerable possibilities for electronic miniaturization.

The preparation of metal inks requires powders with diameter less than 1/100 the size of the nozzle diameter. Since the diameter of the nozzle can be as small as $21 \mu \mathrm{m}$, the powder should be around $200 \mathrm{~nm}$ in size; otherwise nozzle clogging would normally occur.

A significant decrease in particle size increases the difficulty to produce highly concentrated stable dispersions due to the stronger agglomeration, the high density and extremely high surface energy, and therefore it requires the development of new dispersion procedures, including selection of appropriate dispersants and tools to obtain long-term dispersion stability.

Once the stable dispersion of nanoparticles is obtained, the ink formulation must be adjusted according to the given specification regarding the dimension of the patterns as well as required resolution. Additionally the rheological behaviour must be adapted to reach the process requirements. Viscosity should be between 1-12 mPas at operating temperature. The printhead can be heated up to $70^{\circ} \mathrm{C}$ in order to lower the working viscosity in case that the fluid is too viscous. Viscosities up to $30 \mathrm{mPas}$ at jetting temperature have been jetted, but drop velocities may be too slow for some applications and the operating performance is typically limited.

The morphology of the printed lines has an effect on the bulk electrical properties of the printed line [4]. Smaller feature size enables the benefits of inkjet printing in functional applications, such as printed electronics. Additionally, it lowers the production costs of 
electronic devices, since material can be positioned on-demand, which reduces the amount of necessary materials [4].

In order to achieve thick conductive tracks with few printed layers, high solid content has to be loaded in the ink. The more concentrated the ink is, the thicker film is possible to obtain with better conductive properties, which would save time and reduce the costs of the process.

The silver inks described in literature have maximal solid loading of $25 \mathrm{wt} \%[5,6]$. This work reports a silver ink with $45 \mathrm{wt} \%$ solid loading, while remaining the stability and good performance.

Most syntheses of metal nanoparticles (NPs) reported in literature take place in organic mediums, which might be explained by the fact that most capping agents that control the particle size and prevent the agglomeration, are soluble in organic medium $[7,8]$. Nevertheless, the hazardous organic solvents that are used during synthesis produce an array of toxic wastes. For that reason, the environmental - friendly aqueous synthesis of metal nanoparticles and the subsequent use of so prepared material in water-based nanofluids for inkjet printing process are in need of present industry.

The long-term dispersion stability of Ag NPs in an aqueous medium might be achieved through electrostatic repulsion between particles. Anionic surfactant absorbed at particles surface gives rise to electrostatic repulsion. In this study, the surface of synthesized Ag NPs was capped by dioctyl sodium sulfosuccinate (AOT) that also acts as a dispersant in an aqueous medium. The caped Ag NPs were easily dispersed in water up to $25 \mathrm{wt} \%$. However, further increasing the silver content weakens the stability of the fluid. This phenomenon is related to the decrease in the electrophoretic mobility of the particles [9]. To enable further increasing in the silver content in an ink, copolymer F127 was used. This triblock co-polymer A-B-A type, where block B has an affinity for the particles and block A 
is responsible for colloidal stabilisation [10], considerably improves stability of highlyconcentrated Ag NPs suspension.

In our earlier work [11], a method to prepare conductive inks based on silver nanoparticles and the copolymer was reported. In that conductive ink, only $5 \mathrm{wt} \%$ of silver nanoparticles could be dispersed in water. To produce a thick and conductive film many layers must be printed. In the present work, a method of synthesizing a highly concentrated (45 wt $\%$ ) and stable silver ink was reported. The conductive Ag patterns were printed by an inkjet printing on three different tapes/substrates and the influence of ceramic substrate temperature and dot spacing on the morphology of printed patterns was investigated. The resistivity of printed Ag patterns as a function of annealing temperature was also analyzed.

\section{Experimental}

The details of the synthesis and characterization of Ag NPs were described in Ref [11]. However, in this work synthesized Ag NPs were treated with acetone rather than 2propanol as shown in [11].

For the preparation of the Ag conductive ink, Ag NPs were dispersed in DI-water with the addition of copolymer (Pluronic F127). Weight ratio of F127 to Ag particles is fixed at 0.05 . The suspension was treated by high intensity focused ultrasounds (HIFU) technique. The frequency of ultrasound was set at $\sim 75 \mathrm{kHz}$, and the peak intensity of shock wave at 80 MPa. The treated time was fixed at $20 \mathrm{~min}$. Zeta potential of prepared ink was measured with Zetasizer 3000, Malvern Instruments. Thermogravimetric analysis was performed with Setaram Setsys Evolution 16/18 apparatus at a heating rate of $10{ }^{\circ} \mathrm{C} / \mathrm{min}$ in air. The size and morphology of silver nanoparticles and printed patterns were determined using a scanning electron microscopy (S-FEG SEM) on a Philips XL30. The size and distribution of Ag NPs in inks were observed by transmission electron microscopy (TEM) using a Philip CM20 and a 
JEOL JEM 2010F. X-ray diffraction was used to determine the crystal structure of silver particles on a Siemens D5005 diffractometer with $\mathrm{Cu} K_{\alpha}$ radiation and a Goebel mirror. Particle size distribution was determined by nanoparticle tracking analysis (NTA) (LM20, Nanosight).

The tapes/substrates used for inkjet printing in this work are commercial LTCC (low temperature co-fired ceramics) DP951 and CT700 and alumina made by IVF (Sweden).

Inkjet printing was carried out with a DoD Fujifilm Dimatix DMP-2831 ink jet printer. Disposable cartridges (DMC-11610) used in this work are equipped with 16 silicon nozzles located at $254 \mu \mathrm{m}$ spacing; the orifice size of each nozzle is approximately $21 \mu \mathrm{m}$. The ink droplets ejection was achieved by applying a $25 \mathrm{~V}$ pulse lasting $15 \mathrm{~s}$ at a frequency of $1000 \mathrm{~Hz}$. The substrates temperature was varied from 20 to $60^{\circ} \mathrm{C}$.

The contact angle measurement was performed on OCA-Dataphysics and the OWRK method was used to determine surface energy of the surface. The surface roughness was determined by an Altisurf 500 apparatus (INP - Grenobe).

The ink-jet printed silver films were annealed at various temperatures from 250-350 ${ }^{\circ} \mathrm{C}$ for $15 \mathrm{~min}$ in ambient atmosphere to achieve electrical conductivity of the track. The conductivity measurements were performed using a four point probe technique. The electrical resistivity $\rho$ of a printed line was then calculated from:

$$
\rho=R^{*} A / L
$$

were $R$ is the resistance of the line, $L$ its length , and $A$ its cross sectional area, and compared to the value of bulk silver $(1.6 \mu \Omega \mathrm{cm})$.

The geometry of the printed tracks was analyzed with Olympus optical microscope. 


\section{Results and discussion}

In our early work [11], the synthesized Ag NPs were treated by 2-propanol prior to ink preparation. In this study, acetone was used. We observed that acetone-treated Ag NPs could be better dispersed in aqueous-based solvent, probably due to the formation of a more hydrophilic surface. Fig.1 (a) and (b) show the SEM and XRD of synthesized Ag NPs treated with acetone. Ag NPs are well crystallized evidenced by XRD and some agglomerates can be observed in the SEM image, but compared with the particles treated with 2-propanol (IPA) [11], the sizes of these agglomerates are much smaller. Fig.2 (a) shows nanoparticles tracking analysis (NTA) of Ag NPs treated with two solvents. The average size distribution of particle agglomerates treated with acetone is $80 \mathrm{~nm}$ and more uniform compared to those treated with IPA.

The average size of the particles obtained by SEM and TEM pictures is around 30-50 $\mathrm{nm}$. This is smaller than that obtained by NTA analysis (80nm). This is due to the stretching of AOT molecules away from the Ag particles while suspended in a liquid solution, leading to the size measured by NTA is twice as large as the thickness of the AOT layer plus the diameter of the actual particle. On the other hand, for SEM and TEM measurements, the particles are placed on a wafer and the solvent is removed, as a result AOT layer on the particle collapses on its surface. This means that the diameter will decrease compared to that measured by NTA, however it is still bigger than the actual particle size.

However, after the addition of co-polymer F127 the particles reach the size of $10 \mathrm{~nm}$ (Fig.3). This shows the properties of dispersant not only as a stabilizer but also as a comminution aid, which promotes the breakage of the hard agglomerates [12].

The agglomeration state was also determined by the colour of the dispersed nanoparticles in solution. When the diameters of the nanoparticles increased, the maximum absorbance wavelength shifted towards the red which is visually observed in colour of the 
dispersion. It can be seen in the inset of Fig.2 (a) that a dilute solution of Ag NPs treated with acetone presents a yellow-brown colour while the solution with the particles treated with IPA shows a grey colour indicating the presence of bigger agglomerates.

The zeta potentials of two silver aqueous suspensions at different $\mathrm{pH}$ values are presented in Fig.2 (b). In order to generate strong repulsive forces, and assure the suspension being well dispersed and stable, high surface charge density is required. For both silver dispersions, the zeta potential is at the minimum when $\mathrm{pH}$ is between 2 and 3 , indicating that the force of electrostatic repulsion between particles is weaker than it is at other $\mathrm{pH}$ values, and thus precipitation easily occurs. When $\mathrm{pH}$ is in the range of 3 to 8 , the electrostatic repulsion force between particles increases and becomes sufficient to prevent particles from coagulating and settling. At $\mathrm{pH} \sim 8$, the zeta potential shows the highest value which indicates that the electrostatic repulsion force between particles are the strongest so the dispersion stability of $\mathrm{Ag}$ nanoparticles at this $\mathrm{pH}$ is the best. Particles treated with acetone reached a value of zeta potential of $-70 \mathrm{mV}$.

High surface charge density, which generates strong repulsive force and small size of the agglomerates make it possible to formulate highly concentrated and stable inks. However, it was only possible to obtain $25 \mathrm{wt} \%$ Ag loading dispersion through adjusting $\mathrm{pH}$ and further increasing the silver content weakens the stability of the fluid due to the decrease in the electrophoretic mobility of the particles [9]. To load more Ag NPs in an ink and form a stable dispersion, copolymer F127 was used. Fig. 3 shows TEM pictures of silver particles dispersed in water with and without copolymer F127. The primary nanoparticles strongly attract each other through physical forces due to large surface area, immediately forming larger particles. Upon ultrasound treating, the agglomerates were separated and due to the presence of F127, the small particles were prevented from being agglomerated again. It was the surface modification that delayed the agglomeration or clustering. Resulted size of the 
dispersed particles was around $10 \mathrm{~nm}$. As a result, a high Ag loading ink containing $45 \mathrm{wt} \%$ Ag could be produced.

As it was mentioned before, high solid loading in an ink plays important role in building up a thick layer. Fig.4 shows the experimental and calculated thickness of per printed layer against metal loading content. The calculation assumes that an ink droplet forms a spherical cap on the surface (a good assumption from our surface tension studies) with spherical radium $R$, cap radium $r$ and contact angle ( $\theta$ ) (Fig.5). We then assumed that the dot remains at this diameter upon drying and sintering to give a uniform thickness i.e. no coffee stain effect.

Volume of the cap

$$
V=\frac{\pi R^{3}\left(2-3 \sin (90-\theta)+\sin ^{3}(90-\theta)\right)}{3}
$$

Ignoring the added co-polymer and surfactant the drop volume is also given by

$$
V=M_{A g} / \rho_{A g}+M_{\mathrm{H}_{2} \mathrm{O}} / \rho_{\mathrm{H}_{2} \mathrm{O}}
$$

where $M$ and $\rho$ are mass and density respectively.

Also denoting mass loading as $L$

$$
L=\frac{M_{A g}}{M_{A g}+M_{\mathrm{H}_{2} \mathrm{O}}}
$$

Substituting in (2) and re-arranging

$$
M_{A g}=\frac{V}{1 / \rho_{A g}+(1-L) / L \rho_{H_{2} O}}=\pi r^{2} t
$$

where $t$ is thickness of the film.

Based on the Eq. (5), thickness of a printed layer can be calculated if its mass is known and the results of experimental and calculated thicknesses are shown in Fig.4. The 
higher loading the thicker layer can be obtained. $1 \mu \mathrm{m}$ thick layer was obtained using the ink containing $45 \mathrm{wt} \%$ of silver. In practice, the measured thickness is much thicker in the high solid loading region than the calculated thickness. This is because we used the reduced dot spacing $(\leq 20 \mu \mathrm{m})$ leading to the overlapping of adjacent dots while the calculated thickness assumed the lack of such overlapping of adjacent dots. Once the stable and high concentrated dispersion of nanoparticles is obtained, the ink formulation must be adjusted to meet the given specification regarding the dimension of the patterns as well as the required resolution. Since the morphology of the printed lines depends on the interactions between the ink and the substrate, it is important to examine and optimise the printing parameters to achieve desired printed feature. Firstly, it is necessary to examine whether the prepared ink meets inkjet printing requirements. In a piezoelectric inkjet printing process, the generation of drops is based on pressure wave that is electrochemically induced [13]. When a voltage is applied, the piezoelectric material changes shape, which generates a pressure wave in the fluid forcing a droplet of ink to be ejected from the nozzle. That happens only when the kinetic energy of the ink is sufficient to overcome the surface energy interactions.

There are three dimensional numbers, namely, the Reynolds $(R e)$, the Weber $(W e)$ and the $Z$ numbers $(Z)$, which have been proved in recent studies $[14,15]$ to be representative for analysis of a drop formation and to determine the possibility of the fluid to be ejected. The Reynolds number represents a dimensionless ratio of the inertial forces against the viscous stress within a droplet:

$$
\operatorname{Re}=\frac{\rho d v}{\eta}
$$

Where $\rho$ is the density, $d$ the orifice diameter, $v$ the velocity and $\eta$ the viscosity of the droplet. The Weber number is a dimensionless ratio of inertia forces versus the interfacial stress, describing the kinetic-surface energy conversion governed by both the speed of the jet and the surface tension: 


$$
W e=\frac{\rho d v^{2}}{\gamma}
$$

where $\gamma$ is the surface tension of the liquid. Both numbers affect the droplet impact and spreading. Typically for inkjet print process, both the Reynolds and Weber numbers are relatively low and in the order of $1-100$, as the velocity and the size of the droplets are relatively small, which usually prevents splashing of the deposited droplets on the surface.

The Z-number, which is the inverse of the Ohnesorge number $(\mathrm{Oh})$ can be written as a function of both dimensionless $R e$ and We number:

$$
Z=O h^{-1}=\frac{\operatorname{Re}}{\sqrt{W e}}=\frac{\sqrt{\rho d v}}{\eta}
$$

A droplet can be ejected by the DoD printer if $\mathrm{Z}$ is in the range 1-10 and that the droplet volume increases as the value of $Z$ increases. The lower limit $(Z<1)$ is determined by the viscosity and usually high pressure is required to eject a droplet whereas the upper limit $(\mathrm{Z}>$ 10) represents the formation of satellite droplets [16].

The ink characteristics and the calculated dimensionless numbers are summed up in Table 1.

To evaluate the stability of $\mathrm{Ag}$ nanofluids, the viscosity measurement was taken against the time over 20 days (Fig. 6). It was found that there was almost no viscosity change up to 10 days and after this time, viscosity slowly increased with the time. The increase of viscosity from $3.3 \mathrm{mPa}$ s in Day one to $3.5 \mathrm{mPa}$ in Day 20 was due to the agglomeration and the formation of larger particle clusters. However, no sedimentation was observed up to 20 days. The formed large particle clusters due to the agglomeration could be broken and redispersed in the water under the ultrasonic treatment and the rhoelogical properties of the dispersion can resume to the inital values.

The optimized silver ink fulfils all the conditions necessary to achieve a stable and right process with the inkjet printer used in this study. 
For conductive tracks, ideal printed lines are straight and smooth. In order to optimize the electrical performance of the printed features, defaults such as discontinuity of the deposit, spreading of the ink or edge irregularities have to be avoided. Since the behaviour of the ink depends on the interactions between an ink and the substrate, the effect of three different surface characters on the morphology of printed feature was measured. That was done by printing the lines of the Ag ink while varying the number of individual dots that were placed in a line within the span of 1 inch (= dot spacing), and varying substrate temperatures ranging from 23 to $60{ }^{\circ} \mathrm{C}$. The printing parameters used for the experiments were $115 \mathrm{~V}, 35 \mu$ s and $200 \mathrm{~Hz}$.

Fig. 7 presents the final width of the printed lines on different substrates against substrate temperature, at given dot spacing. Increasing the dot spacing reduces the deposited material and hence decreases the width of the printed line (Fig. 8). As a result, it results in smaller printed features. When dot spacing was increased to larger than $60 \mu \mathrm{m}$, partially continuous lines were formed and further increase of the dot spacing led to individual droplets.

The width of the track printed under the same conditions varied with the substrates, e.g. at room temperature the width of the track is $\sim 490,520$ and $680 \mu \mathrm{m}$ for LTCC DP951, alumina and LTCC CT700 respectively while printing with $10 \mu \mathrm{m}$ dot spacing. The different widths of printed lines on different substrates are caused by different surface energies and roughness. The corresponding properties of the substrates are shown in Table 2 and Fig. 9. Table 2 showed that all used tapes possessed high surface energy, around $50 \mathrm{~mJ} / \mathrm{m}^{2}$ for both alumina and LTCC CT700 and $30 \mathrm{~mJ} / \mathrm{m}^{2}$ for LTCC DP951. The surface energy of the ink should be lower than the surface energy of the substrate to perform printing. In all tested substrates the polar contribution of the surface energy is higher than the dispersive one and hence water-based inks are suitable to use (Table 2). Additionally it is also advisable to have 
low surface roughness to promote the ink transfer and obtain the appropriate resolution. A high degree of surface roughness can probably cause an uneven ink distribution and lower print density. Among all three substrates, LTCC CT700 shows the highest surface roughness $(=0.49 \mu \mathrm{m})($ Fig. 9$)$ and on this tape the ink spreads the most (Fig. $7 \& 8)$.

The width of the lines printed becomes narrower with the increase of the substrate temperature (Fig. 7). However the decrease in the line width between $23{ }^{\circ} \mathrm{C}$ and $40{ }^{\circ} \mathrm{C}$ is greater than that between $40{ }^{\circ} \mathrm{C}$ and $60{ }^{\circ} \mathrm{C}$, which may be due to the quicker evaporation of water at higher temperature, resulting in a lower degree of spreading. With increasing the substrate temperature, the width of the track is reduced. As the evaporation rate of water increases it results in the increase of solid concentration in the ink and subsequently the fast increase in viscosity. The increase of viscosity of the silver ink decreases the mobility of the ink droplet and consequently prevents its spreading and ensures the desired print quality. However, for the cases of wider dot spacing, the temperature does not show such a significant influence. This is because the evaporation of droplets occurs fast enough as there is little influence of the interaction with neighbouring dots (Fig.8). However, the increase in temperature didn't help reduce the coffee stain effect, as the shinning edge of printed lines can be seen in Fig. 8, indicating the higher deposit of the material along the edges.

In order to perform resistivity measurement of the printed ink, single line was printed onto alumina and subsequently heated up to $450{ }^{\circ} \mathrm{C}$ on a hot plate. The SEM cross-sectional and top views of silver track (Fig. 10) display a dense silver track that was formed with well packed particles.

The resistance of the printed $\mathrm{Ag}$ lines drastically decreases when annealing temperature increases (Fig. 11). The lowest temperature at which printed features become conductive is mainly determined by the outlet of evaporation of organic additives in the ink. The decomposition temperature (at which the sample has the highest weight loss) was observed to 
be around $250{ }^{\circ} \mathrm{C}$. The weight loss is due to the removal of organic molecules through the decomposition process. The total weight loss of organic content is about $8 \%$. The minimum temperature needed for the printed ink to become a conductive dense film was at $250{ }^{\circ} \mathrm{C}$. The completion of the sintering process was at $350{ }^{\circ} \mathrm{C}$ at which the resistivity $(3 \mu \Omega \mathrm{cm})$ was shown to be only twice higher than bulk Ag. Further heating up to $450{ }^{\circ} \mathrm{C}$ didn't influence the resistivity value. The decrease in resistance depends on the temperature range in which the last part of the organics is burnt off.

The loss of mass does not immediately cause the lines conductive through particles connection. Combining the results of TGA analysis and resistivity measurement it can be observed that half of the organic part is required to be removed to ensure the film to be conductive. All organics have to be removed from the film before it reaches the lowest resistivity.

Additionally, most of the chemicals added during the formulation of inks are acetonesoluble ones. These chemicals can be further removed by washing in acetone and water after the printed patterns are heated at $150{ }^{\circ} \mathrm{C}$. The high conductive track can be obtained too. Therefore the inks can also be applied to the flexible substrates like polymers. The work on Ag ink-jet printing on flexible substrates will be published in another paper.

\section{Conclusion}

Ag NPs with a size of $\sim 10 \mathrm{~nm}$ in aqueous medium were successfully synthesized. An environmental friendly and cost effective method for preparation of high concentrated (45 wt $\%)$ silver - water based ink for the use in a drop-on-demand inkjet printer was presented. Copolymer F127 was found to be very effective as a stabilizer leading to the formation of high silver loading. Prepared ink was inkjet printed on three different ceramic substrates, and highly conductive silver tracks were obtained. The printed patterns showed good shape 
definition. The resistivity of the printed $\mathrm{Ag}$ lines decreases as a function of annealing temperature and it was measured to be $3 \mu \Omega \mathrm{cm}$ after annealing at $350^{\circ} \mathrm{C}$.

\section{Acknowledgments}

Authors would like to thank the European Project (FP7) "MULTILAYER: a large scale production of micro devices via new rolled multi material layered 3D shaping technology", for the financial support with the reference number: FP7-NMP4-2007-214122. Authors also thank Micro Systems Engineering GMbH (Germany) for providing us LTCC tapes and Swerea IVF (Sweden) for $\mathrm{Al}_{2} \mathrm{O}_{3}$ ceramics, Omnisys Instruments for the design of the printed patterns and EFPG (France) for performing surface energy and roughness measurements.

\section{References}

1. L. Hou, F. Huang, W. Zeng, J. Peng, Y. Cao, High-efficiency inverted top-emitting polymer light-emitting diodes. Appl. Phys. Lett., 87 (2005) 1.

2. S.A. Day, D.P. Butler, Z. Celik-Bulter, Micromachined infrared bolometers on flexible polyimide substrates. Sensors Actuators A., 118 (2005) 49.

3. S. Tung, S.R. Witherspoon, L.A Roe, A. Silano, N. Ferraro, A MEMS-based flexible sensor and actuator system for space inflatable structures, Smart Mater. Struct., 10 (2001) 1230.

4. S. Magdassi, The chemistry of silver ink, World Scientific Publishing, Singapore (2010) 246.

5. Sunho Jeong, Hae Chun Song, Won Woo Lee, Youngmin Choi, and Beyong-Hwan Ryu, "Preparation of aqueous Ag Ink with long-term dispersion stability and its inkjet 
printing for fabricating conductive tracks on a polyimide film", J. Appl. Phys., 108(2010), 102805

6. Sunho Jeong, Hae Chun Song, Won Woo Lee, Youngmin Choi, Sun Sook Lee, and Beyong-Hwan Ryu, "Combined Role of Well-Dispersed Aqueous Ag Ink and the Molecular Adhesive Layer in Inkjet Printing the Narrow and Highly Conductive Ag Features on a Glass Substrate", J. Phys. Chem. C, 114 (2010), 22277-22283

7. L. Hsien-Hsueh, Ch. Kan-Sen, H. Kuo-Cheng, Inkjet printing of nanosized silver colloids, Nanotechnology 16 (2005) 2436

8. K. J. Lee, B.H. Jun, T.H. Kim, J. Joung, Direct synthesis and inkjetting of silver nanocrystals toward printed electronics, Nanotechnology 17 (2006) 2424.

9. M. Kaszuba, J. Corbett, F. M. Watson, High-concentration zeta potential measurements using light-scattering techniques, Phil. Trans. R. Soc. A September 28, 2010, 368 (1927) 4439.

10. S-H Ma, C Ford, Block copolymers of oxazolines and oxazines as pigment dispersant and their use in ink jets, (1999), EP0915138.

11. A. Kosmala, R. Wright, Q. Zhang, P. Kirby, Synthesis of silver nano particles and fabrication of aqueous Ag inks for inkjet printing, Mater. Chem. Phys, 129 (2011) 1075.

12. P.C Kapur, T.W. Healy, P.J. Scales, D.Boger, D. Wilson, Role of the dispersant in kinetics and energetic of stirred ball mill. International Journal of Mineral Processing, 47, (1996), 141-152

13. N. Reis, C. Ainsley, B. Derby, Ink-jet delivery of particle suspensions by piezoelectric droplet ejectors, J Appl Phys., 97 (2005) 094903

14. R.M. Meixner, D. Cibis, K. Krueger, H. Goebel, Characterization of polymer inks for drop-on-demand printing systems, Micro-Syst. Technol. 14 (8) (2008) 1137. 
15. V. Fakhouri, G. Mermoud, J.Y. Kim, A. Martinoli, J. Brugger, Drop-on-demand inkjet printing of su-8 polymer, Micro Nanosyst., 1 (2009) 63.

16. K.A. M. Seerden, N. Reis, J. R. G. Evans, P. S. Grant, J. W. Halloran, B. Derby, InkJet Printing of Wax-Based Alumina Suspensions, J. Am. Ceram. Soc., 84 (2001) 2514.

\section{Figure Captions}

Table 1 Calculation of $W e, R e$ and $Z$ dimensionless numbers for the silver ink from ink properties and printer.

Table 2 Surface energies of substrates

Fig.1 SEM (a) and XRD (b) of silver particles.

Fig.2 NTA analysis of silver nanoparticles (a) and zeta potential in dependence on $\mathrm{pH}$ for the nanosilver powders treated with IPA and acetone.

Fig.3 TEM images of silver particles dispersed in water without (a) and with addition of F127 (b).

Fig.4 Thickness per layer for different loading of silver in an ink theoretical (blue line) and experimental (red line).

Fig.5 Schematic graph representing droplet on the surface.

Fig. 6 Viscosity change of Ag nanofluid against time

Fig.7 Width of the printed silver lines at different dot spacing (legend) on (a) CT700, (b) DP951, and (c) alumina substrates against substrate temperatures.

Fig.8 Optical microscope pictures of printed lines at $40{ }^{\circ} \mathrm{C}$ on (a) CT700, (b) DP951and (c) alumina with $10 \mu \mathrm{m}$ dot spacing, and on (d) CT700, (e) DP951 (f) alumina with $40 \mu \mathrm{m}$ dot spacing. 
Fig.9 Roughness analysis results for (a) LTCC CT CT700, Ra $=0.49 \pm 0.07 \mu \mathrm{m}$, (b) LTCC DP951, $\mathrm{Ra}=0.36 \pm 0.01 \mu \mathrm{m}$, and, (c) alumina $\mathrm{Ra}=0.24 \pm 0.07 \mu \mathrm{m}$.

Fig.10SEM pictures of printed silver on $\mathrm{Al}_{2} \mathrm{O}_{3}$; cross-section (a), top view (b) and (c).

Fig.11Resistance over a single inkjet printed line as function of temperature and thermogravimetric analysis (TGA) of silver nanoparticles. 